\title{
Investigating Sexual Function and Factors Affecting It in Women with Breast Cancer in Iran
}

\author{
Fatemeh Zahra Karimi ${ }^{1,}$, Fatemeh Homaee Shandiz ${ }^{2}$
}

${ }^{1}$ Assistant Professor of Reproductive Health, , Department of Midwifery, EvidenceBased Health Care Research Center, School of Nursing and Midwifery, Mashhad University of Medical Sciences, Mashhad, Iran

${ }^{2}$ Department of Radiotherapy, Ghaem Hospital, Radiation Oncology Center Reza, Mashhad University of Medical Sciences, Mashhad, Iran

* Corresponding author: Fatemeh Zahra Karimi, Assistant Professor of Reproductive Health, Department of Midwifery, Evidence-Based Health Care Research Center,

DOI: $10.21859 /$ mci-supp-94 School of Nursing and Midwifery, Mashhad University of Medical Sciences, Mashhad,

Keywords:

Sexual Function

Women

Breast Cancer

Iran Iran.E-mail: karimifz901@mums.ac.ir

\begin{abstract}
Introduction: Breast is strongly relevant to sexual desires, physical and sexual attractiveness, and due to the high prevalence of breast cancer (BC) in Iran and long-term survival of patients with their further involvement in side effects and consequences of BC, taking measures in order to identify their sexual problems seem necessary. Therefore, this study was conducted to assess sexual function and factors affecting it in women with BC.

Materials and Methods: This cross-sectional study was performed on 94 women with BC, referred to Imam Reza (AS) Hospital, Mashhad, Iran, in 2014. Data were collected through demographic and clinical questionnaire and also sexual function questionnaire, then analyzed using SPSS version 16.

Results: Total score of women's sexual function was about $24.34 \pm 4.41 .71 .3$ percent of women participated in this study (63 patients) had sexual dysfunction, while, only 28.7 percent of them (27 patients) had normal sexual function.

Conclusions: Breast cancer can affect women's sexual function and decrease it. Also incidence of sexual dysfunction in women with breast cancer is high, so taking measures to eliminate women's sexual problems are necessary.
\end{abstract}

